\title{
Subwavelength-size solid immersion lens
}

\author{
Myun-Sik Kim, ${ }^{1, *}$ Toralf Scharf, ${ }^{1}$ Mohammad Tahdiul Haq, ${ }^{2}$ Wataru Nakagawa, ${ }^{2}$ and Hans Peter Herzig ${ }^{1}$ \\ ${ }^{1}$ Optics \& Photonics Technology Laboratory, Ecole Polytechnique Fédérale de Lausanne (EPFL), \\ Breguet 2, 2000 Neuchâtel, Switzerland \\ ${ }^{2}$ Electrical and Computer Engineering Department, Montana State University, P.O. Box 173780, \\ Bozeman, Montana 59717-3780, USA \\ ${ }^{*}$ Corresponding author: myunsik.kim@epfl.ch
}

Received July 27, 2011; revised August 25, 2011; accepted September 6, 2011; posted September 9, 2011 (Doc. ID 151715); published September 30, 2011

\begin{abstract}
We report on the fabrication and characterization of nanoscale solid immersion lenses (nano-SILs) with sizes down to a subwavelength range. Submicrometer-scale cylinders fabricated by electron-beam lithography are thermally reflowed to form a spherical shape. Subsequent soft lithography leads to nano-SILs on transparent substrates for optical characterization. The optical characterization is performed using a high-resolution interference microscope with illumination at $642 \mathrm{~nm}$ wavelength. The focal spots produced by the nano-SILs show both spot-size reduction and enhanced optical intensity, which are consistent with the immersion effect. (c) 2011 Optical Society of America

OCIS codes: 220.4241, 310.6628, 180.3170.
\end{abstract}

Hooke first discussed the immersion technique to improve the imaging performance of a microscope in 1678 [1]. Although the concept of homogeneous immersion preceded Abbe's pioneering work, he constructed the first oil-immersion lens in developing the imaging theory of microscopy [2]. Abbe also developed the standard measure of performance of an objective lens, the numerical aperture (NA), which is defined as [3]

$$
\mathrm{NA}=n \cdot \sin \theta,
$$

where $\theta$ is the angle a ray makes with the optical axis (half the angle of the focusing cone), and $n$ is the refractive index of the medium through which the rays pass. In 1990, Mansfield et al. developed a new immersion concept, which is termed solid immersion lens (SIL) [4]. Focusing in the center of a hemispherical solid leads to normal incidence of the rays with respect to the spherical interface. Therefore, there is no refraction at the interface between the spherical solid and the surrounding medium (e.g., air), leading to an increase in the NA by a factor of $n$ (the refractive index of the solid medium) as shown in Eq. (1).

At the beginning, the fabrication of SILs was limited to macroscopic size (i.e., millimeter scale). Advances in micro- and nanofabrication technologies enabled the development of different types of SILs, including diffractive SILs []ㅡ, micrometer-size SILs [ㅁ-]ㅡ, nanoscale spherical lenses [10], and wavelength-scale SILs [11].

The goal of this Letter is to report on the fabrication and optical characterization of nanoscale SILs (nanoSILs) with sizes down to subwavelength range. In general, for structures smaller than the optical wavelength, design methods for larger devices, such as ray optics, are not applicable. More specifically, subwavelength-scale lenses cannot simply be considered to be refractive optical surfaces. However, recent modeling work has shown that subwavelength-scale SILs are still expected to produce a reduced size focal spot [11], the so-called immersion effect. To the best of our knowledge, in this Letter we report the first experimental demonstration of the immersion effect in subwavelength-scale nano-SILs.
Electron-beam lithography (EBL) was used to form cylindrical nanostructures with two dimensions: (1) $600 \mathrm{~nm}$ diameter and $200 \mathrm{~nm}$ height; and (2) $450 \mathrm{~nm}$ diameter and $150 \mathrm{~nm}$ height, which are shown in the scanning electron microscope (SEM) images of Figs. 1(a) and 1(b), respectively. An area of approximately $\overline{4 \mu \mathrm{m}}$ square has been exposed to pattern a cylinder in the center.

Since polymethyl methacrylate (PMMA) is a positive resist, during the development process this area is removed, leaving a free-standing cylinder of PMMA. As PMMA is also a thermoplastic, thermal reflow can be applied to transform a cylinder into a hemispherical shape. To obtain optimal spherical structures, the $600 \mathrm{~nm}$ diameter cylinders are reflowed at $150{ }^{\circ} \mathrm{C}$ for 20 minutes on a hot plate, and the $450 \mathrm{~nm}$ cylinders at $140^{\circ}$ for $20 \mathrm{~min}$ utes. Since the cylinders are fabricated on silicon substrates, the reflowed spherical structures must be replicated on glass substrates for optical characterization in the visible spectrum. Figures 2(a) and 2(b) show SEM images of the replicated spherical structures on glass substrates.

The nano-SIL samples replicated by soft lighography [12] are made of a UV curable polymer (Norland, NOA $65, n=1.52$ ) on borosilicate glass cover slips (thickness $=150 \mu \mathrm{m}, n=1.52$ ). The $600 \mathrm{~nm}$ cylinder was transformed into a spherical cap with a diameter of $700 \mathrm{~nm}$ and a height of $54 \mathrm{~nm}$, which yields a radius of curvature (ROC) of $1.16 \mu \mathrm{m}$, as shown in Fig. 2(a). Figure 2(b) shows the $530 \mathrm{~nm}$ diameter and $45 \mathrm{~nm}$ height spherical cap $(\mathrm{ROC}=781 \mathrm{~nm})$ from the $450 \mathrm{~nm}$ cylinder. During the fabrication process, the volume change and

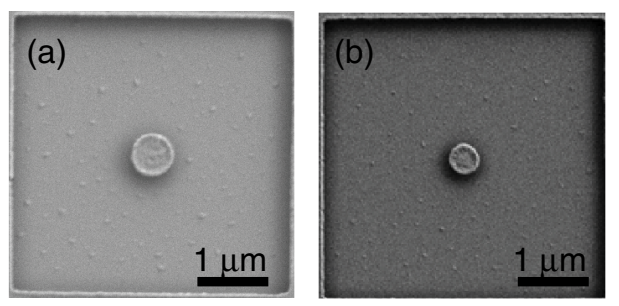

Fig. 1. SEM images of PMMA cylindrical structures on the silicon substrate: (a) diameter $=600 \mathrm{~nm}$ and height $=200 \mathrm{~nm}$ and (b) diameter $=450 \mathrm{~nm}$ and height $=150 \mathrm{~nm}$. 


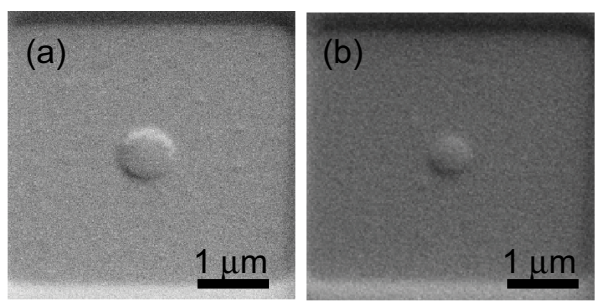

Fig. 2. SEM images of polymer spherical structures replicated on the glass substrate after reflow: (a) diameter $=700 \mathrm{~nm}$ and height $=54 \mathrm{~nm}$ and (b) diameter $=530 \mathrm{~nm}$ and height $=45 \mathrm{~nm}$.

the increase of the diameter led to a decrease in the height, which leads to a subhemispherical SIL [11].

For optical characterization we employ a high-resolution interference microscope (HRIM) to measure the three-dimensional (3D) field distributions of highly confined light fields with NA $\geq 0.9$. The HRIM has already proven to be a powerful tool to characterize micro- and nano-optical elements, such as nanostructures [13], focal spots in optical discs [14], microlenses [15], and photonic nanojets $[16,17]$. Details of the experimental setup are reported in our previous works $[15,17]$. As measurements of field amplitudes are subject to the diffraction limit of the observing objective, we use a 100X/NA1.4 immersion objective (Leica Microsystems, HXC PL APO) in order to obtain the highest resolution in oil immersion. The HRIM is equipped with a light source of wavelength $642 \mathrm{~nm}$ (CrystaLaser, DL640-050-3).

The top row of Fig. 3 shows the charge-coupled device (CCD) camera images of the $700 \mathrm{~nm}$ nano-SIL and the focal spot. The dark point in the center is the SIL and the square is the $4 \mu \mathrm{m}$ square area where the PMMA has been patterned. The focusing lens is a dry objective 50X/NA0.9 HXC PL APO (Leica Microsystems).

In order to verify the immersion effect, we illuminate the sample with a focused beam at three different
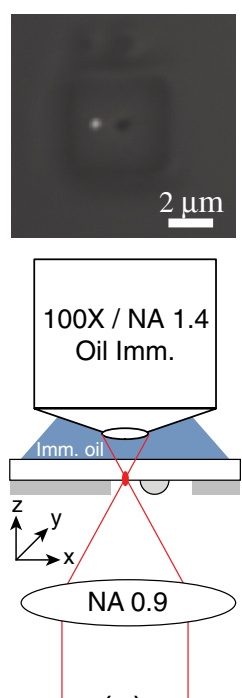

(a)
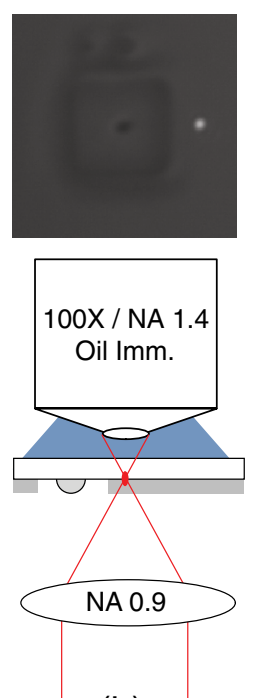

(b)
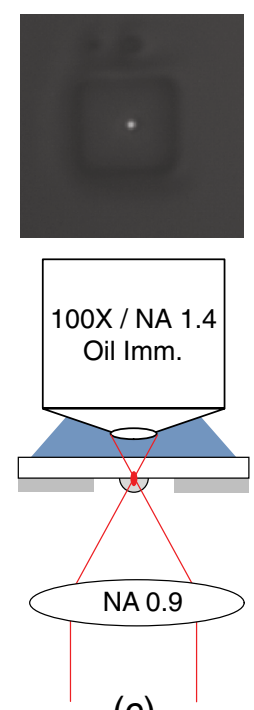

(c)
Fig. 3. (Color online) Geometries of the optical characterization experiments (bottom row) and the corresponding CCD images (top row): focal spot (a) at the interface between air and glass in an area without polymer, (b) at the glass-polymer interface illuminated through a planar polymer, and (c) illuminating the $700 \mathrm{~nm}$ nano-SIL. positions, as schematically illustrated in the bottom row of Fig. 3. First, we focus at the interface between the air and the glass substrate in the exposed region where there is no polymer, as shown in Fig. 3(a). This represents the reference case. Second, we focus on the polymer-glass interface, illuminating through the $200 \mathrm{~nm}$ thick planar region of the polymer, as shown in Fig. 3(b). Comparing these two cases, we confirm there is no immersion effect when the focal spot is projected through the planar interface. Since the depth of the planar layer is only $200 \mathrm{~nm}$, spherical aberration is negligible, and there is practically no degradation of the focus in Fig. 3(b) compared to the case of Fig. 3(a). Third, we focus on the nano-SIL. A comparatively smaller focal spot is observed, as shown in the top image of Fig. 3(c).

To evaluate the reduction of the spot size, we compare the amplitude and phase distributions of the focal spots from the cases of Figs. 3(a) and 3(c) for both $700 \mathrm{~nm}$ and $530 \mathrm{~nm}$ SILs. The transverse and longitudinal cross sections of the $3 \mathrm{D}$ data through the peak intensity points are shown in Fig. 4.

The HRIM yield $\overline{\mathbf{s}}$ both amplitude and phase data for the observed optical fields, and therefore can provide super-resolution (i.e., exceed the diffraction limit). In particular, the lateral position accuracy of specific phase features, such as the position of phase jumps or singularities, are not subject to the diffraction limit [13]. For example, since singularities are mathematically exact points, measuring their distance or position is only limited by the signal-to-noise ratio, and therefore superresolution is generally possible. However, this superresolution does not refer to a physical object but only to particular topological features in the wave field. Therefore, the resolution in the $x-y$ phase plane is in general given by the demagnified size of the pixels from the

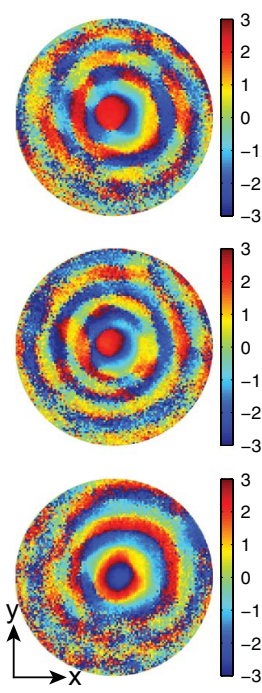

(a)
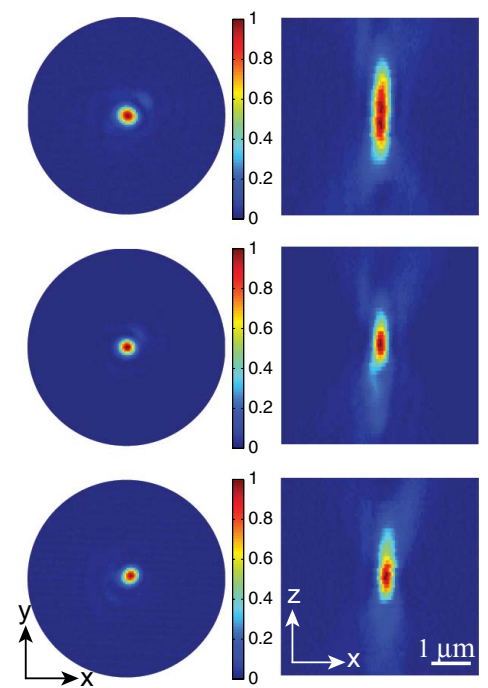

(b)

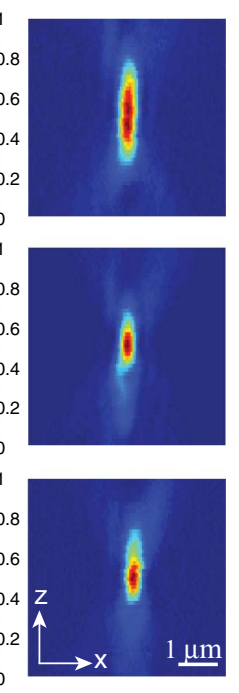

(c)
Fig. 4. (Color online) Cross sections of the 3D fields through the maximum intensity point for (a) transverse phase distributions, (b) transverse and (c) longitudinal intensity distributions of the focal spots. The top row shows the reference focal spot [see Fig. 3(a)], the middle row shows the focal spot produced by the $700 \mathrm{~nm}$ SIL [see Fig. 2(a)], and the bottom row shows the focal spot produced by the $530 \mathrm{~nm}$ SIL [see Fig. 2(b)]. The intensities are normalized. 


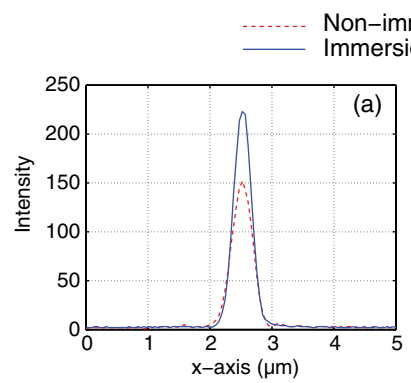

Non-immersion focal spot

Fig. 5. (Color online) Lateral ( $x$ axis) and axial ( $z$ axis) intensity profiles of the reference and nano-SIL focal spots: (a) $x$ axis and (b) $z$ axis profiles. Dashed lines denote the reference focal spot and solid lines denote the focal spot of the $700 \mathrm{~nm}$ nano-SIL.

image (camera) plane to the object plane. The HRIM demonstrated a lateral position accuracy down to $20 \mathrm{~nm}$ for wave fields in our previous work [18]; the current setup provides a $100 \mathrm{~nm}$ accuracy. In this way, the phase fields provide a more precise measure of the spot-size reduction. For the transverse $(x-y)$ phase measurements at the focal plane, the comparison of the size of the central isophase discs, which represent the Airy disc of the intensity distributions $(1.22 \lambda / \mathrm{NA})$, directly shows the reduction of the full spot size rather than the FWHM spot size $(0.5 \lambda / \mathrm{NA})$ in the amplitude measurements.

The focusing objective has an NA of 0.9 , which leads to a predicted Airy disc size of $870 \mathrm{~nm}$ and FWHM spot size of $360 \mathrm{~nm}$ at $642 \mathrm{~nm}$ wavelength. For the reference configuration, the Airy disc size is measured to be $880 \mathrm{~nm}$ and the FWHM spot size is measured to be $400 \mathrm{~nm}$ as shown in the top row of Figs. 4(a) and 4(b), respectively. These results show good agreement with the expected values. The results shown in the middle row of Fig. 4 allow the measurement of the Airy disc (full spot) and the FWHM spot sizes for the $700 \mathrm{~nm}$ nano-SIL, which are found to be $650 \mathrm{~nm}$ and $336 \mathrm{~nm}$, respectively. The equivalent results for the $530 \mathrm{~nm}$ nano-SIL are shown in the bottom row of Fig. 4, and yield results of $660 \mathrm{~nm}$ for the full spot size and $33 \overline{7} \mathrm{~nm}$ for the FWHM.

As discussed above, the super-resolution performance due to the amplitude and phase measurement data provided by the HRIM, allows evaluation of the spot-size reduction of the full spot with an accuracy of $100 \mathrm{~nm}$ in the current setup. The measured reduction ratio (i.e., the reference spot size divided by the nano-SIL spot size) is approximately 1.35 . We assume that the subhemispherical shape [11] of the nano-SIL is the cause of the lower reduction ratio than would be expected given the refractive index of the nano-SIL $(n=1.5)$.

To evaluate the intensity enhancement due to the immersion effect, we compare the lateral and axial intensity profiles of the reference focal spot and the immersion focal spot for the $700 \mathrm{~nm}$ SIL as shown in Fig. 5. The peak intensity is enhanced approximately $50 \%$ by the immersion effect due to stronger spatial confinement of the field.

In conclusion, to the best of our knowledge, we have demonstrated for the first time the fabrication and optical characterization of nanometer-scale SILs down to subwavelength size. The nano-SILs were fabricated by EBL and thermal reflow of the PMMA structures. The measured amplitude and phase distributions from the HRIM showed the reduction of the FWHM spot and the full spot (Airy disc) sizes, respectively. The reduction ratio for the full spot size was found to be 1.35 rather than the refractive index of the SIL of 1.5. The subhemispherical shape is assumed to be the cause of the lower reduction ratio. The enhancement of the peak intensity provides an additional confirmation of the immersion effect in the nano-SIL. Prospective applications of such nano-SILs include near-field focusing and imaging [11], optical data storage [19], and maskless direct-write lithography [20]. Furthermore, the introduction of plasmonic structures on the bottom of the SIL could further improve performance and resolution [21]. Future work will improve the shape of nano-SILs produced using this method.

The research leading to these results has received funding from the European Community's Seventh Framework Programme FP7-ICT-2007-2 under grant agreement No. 224226.

\section{References}

1. R. Hooke, Microscopium (Royal Society, 1678).

2. E. Abbe, J. R. Microsc. Soc. 2, 812 (1879).

3. E. Abbe, J. R. Microsc. Soc. 21, 388 (1881).

4. S. M. Mansfield and G. S. Kino, Appl. Phys. Lett. 57, 2615 (1990).

5. R. Brunner, M. Burkhardt, A. Pesch, O. Sandfuchs, M. Ferstl, S. Hohng, and J. O. White, J. Opt. Soc. Am. A 21, 1186 (2004).

6. D. A. Fletcher, K. B. Crozier, K. W. Guarini, S. C. Minne, G. S. Kino, C. F. Quate, and K. E. Goodson, J. Microelectromech. Syst. 10, 450 (2001).

7. M. Lang, T. D. Milster, E. Aspnes, T. Minamitani, and G. Borek, Jpn. J. Appl. Phys. 46, 3737 (2007).

8. T. Kishia, S. Shibata, and T. Yano, J. Non-Cryst. Solids 354, 558 (2008).

9. M. Brun, M. Richard, and S. Nicoletti, International Symposium on Optical Memory (ISOM09), Mo-E-04 (2009).

10. J. Y. Lee, B. H. Hong, W. Y. Kim, S. K. Min, Y. Kim, M. V. Jouravlev, R. Bose, K. S. Kim, I. C. Hwang, L. J. Kaufman, C. W. Wong, P. Kim, and K. S. Kim, Nature 460, 498 (2009).

11. D. R. Mason, M. V. Jouravlev, and K. S. Kim, Opt. Lett. 35, 2007 (2010).

12. Y. Xia and G. M. Whitesides, Annu. Rev. Mater. Sci. 28, 153 (1998).

13. C. Rockstuhl, I. Märki, T. Scharf, M. Salt, H. P. Herzig, and R. Dändliker, Current Nanosci. 2, 337 (2006).

14. M.-S. Kim, T. Scharf, and H. P. Herzig, Jpn. J. Appl. Phys. 49 , 08KA03 (2010).

15. M.-S. Kim, T. Scharf, and H. P. Herzig, Opt. Express 18, 14319 (2010).

16. M.-S. Kim, T. Scharf, S. Mühlig, C. Rockstuhl, and H. P. Herzig, Appl. Phys. Lett. 98, 191114 (2011).

17. M.-S. Kim, T. Scharf, S. Mühlig, C. Rockstuhl, and H. P. Herzig, Opt. Express 19, 10206 (2011).

18. C. Rockstuhl, M. Salt, and H. P. Herzig, J. Opt. A 6, S271 (2004).

19. B. D. Terris, H. J. Mamin, and D. Ruger, Appl. Phys. Lett. 65, 388 (1994).

20. L. P. Ghislaina, V. B. Elings, K. B. Crozier, S. R. Manalis, S. C. Minne, K. Wilder, G. S. Kino, and C. F. Quateb, Appl. Phys. Lett. 74, 501 (1999).

21. Y. Kim, S. Kim, H. Jung, E. Lee, and J. W. Hahn, Opt. Express 17, 19476 (2009). 